\title{
Cateter Venoso Central de Inserção Periférica: limites e possibilidades
}

\author{
Peripherally Inserted Central Venous Catheters: limits and possibilities
}

Catéter Venoso Central de Inserción Periférica: límites y posibilidades

Jaqueline Petry ${ }^{1}$, Kelen Tortelli da Rocha² ${ }^{2}$ Adriana Ribeiro Martins Madalosso ${ }^{3}$, Rejane Maria Agne de Carvalho ${ }^{4}$, Marina Scariot ${ }^{5}$

\section{RESUMO}

O presente estudo objetivou identificar limites e possibilidades de expansão do uso do Peripherally Inserted Central Catheters (PICC) em unidades neonatais e pediátricas para outras unidades de internação. Trata-se de uma pesquisa exploratório-descritiva de abordagem qualitativa. A coleta de dados ocorreu em agosto e setembro de 2010, por meio de entrevista semiestruturada, com dez enfermeiros de um hospital do Rio Grande do Sul. A análise dos dados resultou em duas categorias: fatores limitadores da expansão do uso do PICC - falta de conhecimento técnico de profissionais de saúde, elevado custo do procedimento, enfrentamento dessa inovação tecnológica e a especificidade desse recurso - e fatores facilitadores para a expansão do PICC - benefícios para o paciente, diminuição do estresse da equipe e a otimização do tempo de trabalho. Conclui-se que a produção de boas evidências sobre o uso do PICC podem auxiliar os profissionais e as instituições utilizarem melhor esse recurso, beneficiando a qualidade da atenção.

Descritores: Enfermagem Pediátrica; Enfermagem Neonatal; Cateterismo Venoso Central.

\section{ABSTRACT}

The present study aimed at identifying limits and possibilities of expansion in the use of Peripherally Inserted Central Catheters (PICC) in neonatal and pediatric units to other hospitalization units. It consists of an exploratory-descriptive study with a qualitative approach. Data collection took place between August and September of 2010, through a semi-structured interview with ten nurses from a hospital located in Rio Grande do Sul. Data analysis resulted in two categories: limiting factors for the expansion in the use of PICCs - health professionals' lack of technical knowledge, high cost of the procedure, confrontation of this technological innovation and specificity of this recourse - and facilitating factors for the expansion of the PICC, which were - benefits for the patient, decrease of stress for the team and optimization of work time. The authors concluded that the production of sound evidence regarding the use of PICCs may help professionals and institutions utilize this resource more effectively, bringing benefits to healthcare quality.

Descriptors: Pediatric Nursing; Neonatal Nursing; Catheterization, Central Venous.

\section{RESUMEN}

Se objetivó identificar límites y posibilidades de expansión del uso del Peripherally Inserted Central Catheter (PICC) en unidades neonatales, pediátricas y para otras unidades de internación. Investigación exploratorio-descriptiva, cualitativa. Datos recolectados de agosto a setiembre de 2010, mediante entrevistas semiestructuradas con diez enfermeros de un hospital de Rio Grande do Sul. El análisis de datos determinó dos categorías; factores limitantes de la expansión del uso del PICC: desconocimiento técnico de profesionales de salud, alto costo del procedimiento, utilización de la innovación tecnológica y especificidad del recurso; y factores facilitadores para la expansión del PICC: beneficios para el paciente, disminución del estrés del equipo y optimización del tiempo de trabajo. Se concluye en que la producción de evidencias positivas sobre el uso del PICC puede ayudar a profesionales e instituciones a utilizar mejor dicho recurso, incrementando la calidad de la atención brindada.

Descriptores: Enfermería Pediátrica; Enfermería Neonatal; Cateterismo Venoso Central.

\footnotetext{
${ }_{1}^{1}$ Acadêmica do curso de graduação em Enfermagem da Universidade de Passo Fundo (UPF). Passo Fundo, RS, Brasil. E-mail: jakepetry@yahoo.com.br.

${ }^{2}$ Acadêmica do curso de graduação em Enfermagem da UPF. Passo Fundo, RS, Brasil. E-mail: ktortelli@hotmail.com.

${ }^{3}$ Enfermeira, Mestre em Enfermagem. Professora Assistente III da UPF. Passo Fundo, RS, Brasil. E-mail: adrianamadalosso@upf.br.

${ }^{4}$ Enfermeira, Mestre em Enfermagem. Professora Titular da UPF. Passo Fundo, RS, Brasil. E-mail: rejanecarvalho@upf.br.

${ }^{5}$ Acadêmica do curso de graduação em Enfermagem da UPF. Passo Fundo, RS, Brasil. E-mail: marinascariot@hotmail.com.
} 


\section{INTRODUÇÃO}

Durante boa parte do século XIX a saúde das crianças não era tão valorizada pelos profissionais da saúde e as taxas de mortalidade infantil eram altas ${ }^{(1)}$. As crianças hospitalizadas eram tratadas com uma visão centrada nas necessidades biológicas aparentes e na sua patologia, mantidas em isolamento, longe da família(2). Esta realidade mudou a partir dos anos 80 , quando a enfermagem começou a implantar o processo de humanização no cuidado da criança considerando os aspectos emocionais, sociais, familiares e atraumáticos com o uso de novas tecnologias (3-4).

A evolução da enfermagem relacionada aos procedimentos endovenosos se intensificou e se aperfeiçoou no período entre 1980 e 2000(5). A administração de medicações endovenosas faz parte da assistência de enfermagem e é necessário o desenvolvimento de habilidades e conhecimentos que garantam a eficácia da terapia, incluindo a utilização de estratégias de cuidado, entre elas, os cateteres endovenosos como o Peripherally Inserted Cental Catheters (PICC) $)^{(6)}$.

O PICC é um cateter venoso central de inserção periférica para uso de terapia intravenosa e foi descrito pela primeira vez na literatura pelo médico alemão Forssmann, em 1929. Os materiais utilizados eram precários, fazendo com que o procedimento não fosse implementado naquela época. Na década de cinquenta, estudos comprovaram que a infusão de fluidos endovenosos aplicados por cateter de localização central, teria mais eficácia que por acesso periférico. Na década de setenta, se desenvolveram cateteres de silicone e, graças a esse avanço, em 1980 o uso desse dispositivo passou a ser mais utilizado. O Brasil faz uso dessa tecnologia desde os anos noventa ${ }^{(5,7)}$. Este cateter pode ser de poliuretano ou silicone, pois ambos são biocompatíveis e menos trombogênicos, o que dificulta a colonização de microorganismos. Esse cateter pode ser empregado em terapias de médio a longo prazo(5-7).

Em se tratando de crianças, especialmente neonatos, é essencial dispor de um acesso venoso seguro e funcional para a sobrevivência desses pacientes $^{(8)}$. A frequência de acessos intravenosos periféricos favorece a ocorrência de complicações como o extravasamento, que é o escape de sangue ou outro fluído de um vaso sanguíneo para o tecido circundante; a infiltração, caracterizada pela administração inadvertida de uma solução e ou medicamento não vesicante no tecido circunjacente e a flebite, que é inflamação da parede venosa. Esses agravos estão ligados à falta de cuidados assépticos, tipo de infusão, o local da punção, número de punções, entre outros. Esses fatores podem influenciar o período de internação, que pode se prolongar, além do desconforto e dor que causa na criança, podendo interferir no seu crescimento e desenvolvimento ${ }^{(4,6,690)}$.

A necessidade de aperfeiçoar os conhecimentos de enfermagem, no sentido de estar qualificando a assistência, faz com que se busque e se incorpore cada vez mais novas tecnologias de cuidado. Nesse sentido, o PICC veio somar, sendo incorporado à assistência de enfermagem, para contribuir na melhoria da qualidade de vida do paciente pediátrico(11-12).

O local de inserção mais comum é a veia basílica, pelas características anatômicas favoráveis, seguida da veia cefálica. Sua localização deve ser em nível central, ou seja, no terço distal da veia cava superior ${ }^{(4,13)}$.

Entre as indicações do PICC está a manutenção de acesso venoso para terapia de longa e média permanência, a administração de soluções hiperosmolares, vesicantes e irritantes, antibioticoterapia por mais de sete dias, infusão de sangue e hemoderivados ${ }^{(6,11,13)}$. O PICC é contra-indicado para a administração de medicações "em bolus", quando o paciente faz uso de marcapasso cardíaco interno, na presença de lesões cutâneas no local ou próximo da inserção do cateter e nas alterações anatômicas ou estruturais da rede venosa ${ }^{(11,14-15)}$.

Esse tipo de acesso apresenta inúmeras vantagens para recém-nascidos, crianças, equipe e instituição. Dentre as vantagens pode-se citar a preservação da rede venosa, menor risco de infecções, menor restrição da mobilidade, diminuição da dor e desconforto, inserção segura, realização no próprio leito do paciente. Além disso, contribui para a minimização da manipulação necessária ao emprego da técnica, diminuição do tempo e do estresse da equipe, do paciente e familiar pelas punções repetidas, maior relação custo benefício e, pode permanecer instalado no período de até um ano. Quanto às desvantagens, pode-se citar a necessidade de treinamento do profissional e o tempo despendido para o procedimento que envolve em média, quarenta e cinco minutos à uma hora. Após o procedimento, é necessária 
a realização de radiografia para a confirmação da posição central do cateter ${ }^{(9,11,13-16)}$.

No Brasil, a atribuição de competência técnica e legal para o enfermeiro inserir e manipular o PICC, encontra-se amparada pelo Conselho Federal de Enfermagem (COFEN) pela Resolução n 258/2001, que determina ser de competência do enfermeiro a inserção do PICC, desde que qualificado para tal(16-17).

Apesar dos benefícios, este procedimento envolve certa complexidade técnica profissional e, por isso tem sido tradicionalmente empregada em unidades de terapia intensiva e com pacientes neonatais e lactentes jovens. No entanto, o fato deste tipo de pacientes estarem internados em unidades de menor complexidade, não exclui a condição de serem submetidos a punções venosas de repetição e por períodos prolongados com consequente desconforto, dor e comprometimento da rede vascular.

Assim, com a visão da importância desse aparato tecnológico para a melhoria da assistência aos pacientes neonatais e pediátricos e, para facilitar os cuidados da enfermagem, é que o grupo de autores se propôs a desenvolver esse estudo, com o objetivo de investigar os limites e as possibilidades de expansão do uso do PICC das unidades neonatais e pediátricas de cuidados intensivos para outras unidades de internação.

\section{METODOLOGIA}

Trata-se de uma pesquisa do tipo exploratório descritiva, de abordagem qualitativa, que teve o objetivo de identificar os limites e as possibilidades de expansão do uso do PICC das unidades de cuidados intensivos neonatais e pediátricos para outras unidades de internação.

A coleta ocorreu nos meses de agosto e setembro de 2010, com horário e local definidos previamente em comum acordo com as autoras da pesquisa e entrevistados, sem que houvesse interferência nas atividades de trabalho e visando manter os princípios éticos. A pesquisa foi realizada com enfermeiros que atuam nas Unidades de Internação Pediátrica, Centro de Tratamento Intensivo Neonatal (CTIN), Centro de Tratamento Intensivo Pediátrico (CTIP) e Centro de Tratamento Intensivo Intermediário (CTII) de um hospital do interior do Rio Grande do Sul. Este município está localizado no Planalto Médio, no norte do estado e tem uma população estimada de 180 mil habitantes.
O campo do estudo se caracteriza por ser um dos maiores hospitais do município e do estado. É um hospital geral, de ensino e de grande porte, atende várias especialidades, inclusive as de alta complexidade, está credenciado pelo Sistema Único de Saúde (SUS). As unidades onde o estudo foi desenvolvido abrangem um total de 92 leitos, sendo 36 de intensivismo neonatal e pediátrico e 56 de internação pediátrica.

Os participantes do estudo foram enfermeiros que atuam nos turnos da manhã, tarde e noite nas unidades onde foi realizado o estudo. Dos 15 profissionais que compõem esse grupo, participaram 10 deles, sendo o critério adotado para a definição desse número a saturação das falas. Para a coleta dos dados, os participantes tiveram conhecimento do objetivo do estudo e aceitaram participar da pesquisa mediante a assinatura do Termo de Consentimento Livre e Esclarecido.

A técnica empregada para a coleta de dados foi entrevista individual semiestruturada contendo questões relacionadas ao profissional como a idade, o sexo, o tempo de formação, o setor que atua e há quanto tempo, a habilidade para passagem do PICC e há quanto tempo. Em um segundo momento se questionou sobre os fatores que limitam e possibilitam a expansão do uso do PICC de unidades neonatais e pediátricas de cuidados intensivos para outras unidades de internação, além do questionamento sobre os benefícios da utilização desse dispositivo para os pacientes e para os profissionais de enfermagem.

As informações foram gravadas, com autorização do entrevistado, pois esse método permite que as falas dos participantes sejam transcritas com fidedignidade, de forma cuidadosa e sistemática. Posteriormente, as gravações foram destruídas mantendo os princípios éticos do anonimato. Para atender estes requisitos, foram observadas as diretrizes da Resolução 196/1996 do Conselho Nacional de Saúde do Ministério da Saúde para pesquisa que envolve seres humanos.

Os dados coletados foram analisados, buscando identificar os conteúdos emergentes relevantes aos objetivos propostos no estudo(18). A primeira etapa constou da transcrição fiel das gravações e leitura minuciosa do material, com o objetivo de identificação de núcleos de sentido. Numa segunda etapa realizou-se leitura em profundidade e análise para categorização dos mesmos. 
Aos enfermeiros participantes, no intuito de garantir seu anonimato, foram designados códigos para identificação das falas com a utilização da letra E seguido de um número ordinal, conforme a ordem da entrevista.

A pesquisa foi aprovada pelo Comitê de Ética da Universidade de Passo Fundo (CAAE $n^{\circ}$ 0154.0.398.00010) após o consentimento do enfermeiro responsável pelo setor e da autorização da instituição hospitalar.

\section{RESULTADOS E DISCUSSÃO}

Dos 10 enfermeiros que participaram do estudo nove eram do sexo feminino, com idades entre 23 e 38 anos. O tempo de formação variou entre dois meses e 15 anos. Em relação ao setor de trabalho, dois atuavam na Unidade de Internação Pediátrica e os demais nas CTIs Neonatal, CTI Intermediária e CTI Pediátrica.

No que se refere à realização de curso de capacitação para a passagem do PICC, que dá amparo legal ao enfermeiro para a realização do procedimento, e o tempo que o profissional havia sido capacitado, apenas dois enfermeiros não tinham essa formação, pois ambos atuavam há pouco tempo no setor. Os demais já estavam habilitados num tempo que variava de dois meses até oito anos.

A partir da análise das falas se buscou agrupar os relatos que trazem os fatores emergentes mediante as realidades vivenciadas no cotidiano de trabalho dos enfermeiros e se identificou duas categorias: fatores limitadores da expansão do uso do PICC e fatores facilitadores para a expansão do seu uso. A primeira categoria se refere às questões limitadoras que envolvem a especificidade do cateter, o desconhecimento deste recurso pelos profissionais, o custo financeiro elevado e enfrentamento de uma inovação tecnológica. A segunda categoria, se refere aos fatores que facilitam o uso e expansão do uso do PICC que foram em relação ao paciente por diminuir a dor, desconforto e infecções e em relação a equipe pela possibilidade da redução do estresse, pois o uso do cateter diminui a repetição de punções e proporciona uma otimização do tempo da enfermagem.

\section{Fatores limitadores da expansão do uso do PICC}

Os enfermeiros apontaram como um dos fatores que limitam o uso do cateter, a sua especificidade, ou seja, tem uso limitado a uma determinada população, tem indicações específicas, entre outras peculiaridades.
O desconhecimento sobre esse recurso por outros profissionais da área da saúde, inclusive profissionais da equipe médica, que não estão familiarizados com o emprego dessa tecnologia, também foi referido como um fator limitador do uso. Essa condição leva esses profissionais a não fazer uso do PICC por não dominarem suas indicações e cuidados, entre outras implicações. Essas limitações podem ser identificadas nas falas de E1, E2 e E8.

A gente sente bastante receio com o manuseio e com $o$ cuidado do cateter, (...) o medo do cuidado, desconhecido.(E1).

Eu acho que precisa ter mais treinamentos, os enfermeiros devem estar se especializando, e até onde eu sei é só quem trabalha em CTI pediátrica ou neonatal, onde é mais difundido o PICC. (E2).

Falta de conhecimento médico, não sabem que existe. (E8).

A falta de conhecimento do PICC e a limitação de capacitação dos profissionais são considerados entraves para o uso e expansão dessa tecnologia, tornando-a limitada. Observa-se que geralmente apenas os enfermeiros que atuam em UTI pediátrica e neonatal são os que tem conhecimento e domínio deste recurso(19). Desta forma, quando a criança recebe alta destas unidades, poderá existir dificuldades nos cuidados de manutenção ou de novas inserções quando necessárias.

Outro fator citado pelos entrevistados, como limitante, foi a questão financeira que envolve o procedimento. A capacitação dos profissionais deve ser autofinanciada ou fornecida pela instituição empregadora. Ainda, o valor relativamente elevado para a aquisição do cateter e a realização do procedimento implicam em custos.

...a questão financeira também acaba que pesa um pouquinho, e o paciente pediátrico fica mais limitado, o neo se limita ainda mais só com cateter umbilical (E4).

...eu acho que a questão financeira também pesa um pouquinho, para ser liberada a passagem do PICC para outros setores... (E1).

Muitas vezes, a realização da capacitação implica em deslocamento dos profissionais para outras instituições que oferecem esse recurso, além da dificuldade de sua 
substituição no ambiente de trabalho por outro colega capacitado.

Eu acho que a PICC na verdade, o que limita talvez a capacitação é a própria instituição de capacitar todos os profissionais.(E7)

Em relação ao custo do cateter, pode-se considerar que o custo total de uma punção venosa periférica é de US\$ 32,00 e o custo para a inserção do PICC é de US\$ 200,00, o que aparentemente demonstra ser mais caro. Mas se for considerado o tempo em que as crianças precisam de terapia endovenosa, geralmente por mais de sete dias ou de terapia prolongada, terá acesso venoso difícil de se manter podendo assim ser submetida à tentativa de várias punções venosas tendo o custo final igual ao do PICC(17). $^{(17)}$

... por mais que ela tenha um valor bem alto, mas se for ficar picando o paciente todo dia ou todo o turno, no final do tratamento vai dar elas por elas, acho que diminui o custo... (E3)

Esse relato corrobora com alguns resultados encontrados na literatura, que mostram o desconhecimento de profissionais em relação ao uso do cateter, assim como

da relação custo beneficio favorável de um tratamento que tenha indicação do $\operatorname{PICC}(16,19)$.

Nesse enfoque, E1 traz sua vivência sobre a limitação financeira como dificuldade para o uso do PICC em outros setores quando relata:

... a UTI cárdio mostrou interesse, também queria passar, a administração não deixou, acho que em função do custo. (E1)

Ainda, a literatura traz que a indicação do PICC em cardiologia diminui o risco de flebites e o estresse devido a várias punções venosas. Também existe viabilidade no uso do PICC em unidades de transplante de medula óssea, devido ao tempo de permanência do cateter e para mensurar a pressão venosa central(17). Nesse enfoque, pode-se dizer que os benefícios trazidos pelo PICC devem fazer parte da assistência de todos os clientes com indicação do uso. Se a tecnologia pode ser oferecida e se mostrou eficiente, pela maior segurança e por ser menos traumática para os pacientes, é dever moral dos profissionais e instituições de saúde, colocar à disposição para o uso, assim como incentivar o aperfeiçoamento dos profissionais e do procedimento(19).

Ainda foi destacado pelos sujeitos do estudo como limitante, a situação de enfrentamento de uma inovação tecnológica como o PICC.

Eu fui, na verdade, a primeira enfermeira da CTI pediátrica que passou PICC, então tive uma resistência de sair do meu trabalho para fazer um curso para aprender...eu fui liberada mas depois eu tive que pagar essas horas, quando eu voltei e quis começar, o hospital não tinha muito interesse, ai tinha médico que achava que eu estava me metendo ... nem sabiam o que era PICC, mas tivemos sorte quando passamos o primeiro PICC, porque ... durou três meses. (E1)

Estudo realizado mostrou que a escassez de profissionais para a inserção do PICC é o segundo motivo que limita a expansão do seu uso, perdendo apenas para a falta de conhecimento dos profissionais(17). Pode-se inferir que as instituições de saúde ainda não fazem um grande investimento em capacitação dos profissionais por não terem um real conhecimento dos benefícios do procedimento, como, maior durabilidade do acesso venoso, maior economia em termos de tratamento e média de tempo de internação, bem como, o conforto para o paciente e a menor intensidade de manuseio. Essa economia e benefícios agregam inclusive, o menor tempo despendido pelo profissional em realizar repetidas punções, além de evitar perdas de materiais utilizados neste tipo de procedimento e que acabam se tornando mais onerosos pela pouca durabilidade.

\section{Fatores facilitadores para a expansão do uso do PICC}

Os fatores que facilitam a expansão do uso do PICC se consolidam neste estudo, por unanimidade, nas vantagens do uso deste dispositivo para o paciente, o que pode ser identificado nas falas a seguir:

Menos dor, eles teriam mais mobilidade, eu vi assim, bastante na pediatria que as crianças, elas ficam, assim, não digo amarradas, mas ficam com aquelas talas, então elas não podem se movimentar muito para não perder o acesso, então menos dor, mais mobilidade, eu acho.(E2) 
...o principal é o baixo risco de infecção... acho que precisam ser menos picados podendo usar drogas que não podem ser usados num cateter normal.(E4)

...comodidade (...) tanto da equipe como do paciente também. O paciente não vai ser submetido a tantas punções, as punções estressam tanto o profissional, que as vezes não é na primeira, assim como estressa muito mais o paciente que vai ter dor. (E7)

O manuseio excessivo da criança, pelo número de punções repetitivas causam mudanças físicas, comportamentais e emocionais. Um acesso central pode diminuir significativamente essas complicações, além de ser um acesso seguro, confiável e que permite a utilização de soluções hipertônicas com mais segurança(5).

Também pode se observar que as vantagens descritas pelos enfermeiros nas falas de E8 e E10 são muito similares as referenciadas em estudos ${ }^{(14-15)}$ que trazem que o PICC proporciona menos desconforto e dor, redução do estresse do paciente, diminuição do estresse da equipe pelas punções repetitivas e otimização do tempo.

Assim, facilita para nós por causa do tempo, pega e passa uma PICC e ela vai durar dias, meses...(E8)

...otimizar o tempo de trabalho, porque a gente acaba perdendo muito tempo puncionando veia, outro dia mesmo eu passei à tarde inteirinha puncionando, (...) eu podia estar usando o tempo para fazer outro tipo de cuidado...(E10)

Especificamente em relação a questão do tempo profissional, os enfermeiros relataram que 0 despendido para realizar inúmeras punções poderia ser reduzido com a passagem do PICC, otimizando assim, o tempo para a prestação de outros cuidados ao paciente, o que contribuiria para a melhoria da qualidade da assistência, a diminuição do estresse da equipe e a redução do índice de infecções, pois uma vez instalado, o cateter poderá permanecer até o final do tratamento, se observados os cuidados para sua manutenção(19).

\section{CONSIDERAÇÕES FINAIS}

O PICC é uma tecnologia de ponta, inovadora, e que traz benefícios para os pacientes e para as equipes que fazem uso desse aparato tecnológico. Observa-se que a ampliação do uso desse cateter encontra-se limitada, muitas vezes devido a custos, por falta de conhecimento técnico-científico, por dificuldades de alguns profissionais de enfrentar uma nova tecnologia, entre outras. Essas limitações podem interferir no processo de cuidar e na qualidade de vida do paciente, pois é um recurso que está disponível, mas nem sempre utilizado.

Este estudo procurou identificar os limites e as possibilidades de expansão do uso do PICC das unidades de cuidados intensivos neonatais e pediátricos para outras unidades de internação.

A expansão do uso do PICC está em lento processo, necessitando de impulsos, com estudos e pesquisas que demonstrem seus benefícios para se tornar parte das rotinas assistenciais ao paciente. Entre esses benefícios incluem-se a promoção do bem estar e a redução de episódios de dor e possíveis infecções por excesso de manuseio como constatado no presente estudo. Outra situação é em relação ao custo do cateter que a princípio pode parecer elevado, mas se for levado em consideração os inúmeros acessos a que são submetidos às crianças nas internações com dispositivos de menor custo mas de menor durabilidade, o valor poderá ser até maior nesta segunda opção.

Observou-se que apesar de suas vantagens, ainda há muita resistência por parte das instituições e profissionais, dificultando a disseminação desse cateter.

Assim é fundamental o desenvolvimento de estudos que mostrem evidencias sobre a eficiência do uso desse dispositivo para subsidiar a prática de profissionais da saúde, bem como estimular que instituições hospitalares disponibilizem o PICC, pelos seus benefícios ao longo de um tratamento.

\section{REFERÊNCIAS}

1.Rodrigues RG, Oliveira ISC. Os primórdios da assistência aos recém-nascidos no exterior e no Brasil: perspectivas para o saber da enfermagem na neonatologia (1870-1903). Rev. Eletr. Enf. [Internet]. 2004 [cited 2012 abr 26]; 6(2):286-291. Available from: http://www.fen.ufg.br/fen_revista/revista6_2/primordio.html.

2. Schimitz, EM. A enfermagem em pediatria e puericultura. São Paulo: Atheneu; 2000.

3.Collet $N$, Rocha SMM. Transformações no ensino das técnicas em enfermagem pediátrica. $2^{\mathrm{a}}$ ed. Goiânia: AB; 2001.

4. Hockenberry M, Wong DL. Fundamentos de enfermagem

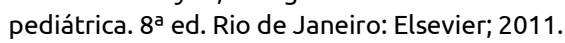


5. Motta PN, Fialho FA, Dias IMAV, Nascimento L. Cateter central de inserção periférica: o papel da enfermagem na sua utilização em neonatologia. HU Rev. 2011;37(2):163-168.

6. Stocco JG, Crozeta K, Labronici LM, Maftum MA, Meier MJ.

Cateter central de inserção periférica: percepções da equipe de enfermagem. Cogitare Enferm. 2011;16(1):56-62.

7. Jesus VC, Secoli SR. Complicações acerca do cateter venoso central de inserção periférica (Picc). Cienc Cuid Saude.

2007;6(2):252-260.

8. Montes SF. Teixeira JBA. Barbosa MH. Barichello E.ocorrência de complicações relacionadas ao uso de cateter venoso central de inserção periférica (PICC) em recém-nascidos. Rev. Eletr. Enf. [Internet]. 2011 [cited 2012 ago 27];

9. Matte MM, Fortes LFF, Pomatti DM, Bettinelli LA. Doenças

Crônicas: possibilidades de cuidados. In: Scariot M, Petry J,

Madalosso ARM, Carvalho RMA. Cateter Venoso Central de Inserção

Periférica: inovação em cuidados de enfermagem, tendências e desafios. Passo Fundo: Berthier; 2011.

10. Gomes AVO, Antunes JCP, Araújo MC, Nascimento MAL. Punção venosa pediátrica: análise crítica de um procedimento de enfermagem. Enferm. Glob. [Internet].

2011[cited 2012 Jun 08]; 10(23):277-286. Available from: http://dx.doi.org/10.4321/S1695-61412011000300019. 11. Chaves EMC, Câmara SMC, Araujo KRC, Feitosa TLO, Bezerra FSM de, Queiroz MVO. Cateter central de inserção periférica: protocolo para recém-nascidos. Rev. Nursing. 2008;11(120):230-4.

12. Rodrigues ZS, Chaves EMC, Cardoso MVLML. Atuação do enfermeiro no cuidado com o cateter central de inserção periférica no recém-nascido. Rev. Bras. Enferm. [Internet]. 2006; [cited 2012 ago 21]; 59(5):626-9. Available from:

http://dx.doi.org/10.1590/S0034-71672006000500006. 13. Kalinowski CE (org.). PROINF: Saúde da Criança e do Adolescente. $2^{\mathrm{a}}$ ed. Porto Alegre: Artmed, 2007.

14. Montes SF, Teixeira JBA, Barbosa MH, Barichello E. Ocorrência de complicações relacionadas ao uso de cateter venoso central de inserção periférica (PICC) em recém nascidos. Enferm.

glob. [Internet]. 2011 [cited 2012 Jun 08];15(24). Available from: http://dx.doi.org/10.4321/S1695-61412011000400001.

15. Vendramin P, Pedreira MLG, Peterlini MAS. Cateteres centrais de inserção periférica em crianças de Hospitais do Município de São Paulo. Rev. Gaúcha Enferm. 2007; 28(3):331-9.

16. Conselho federal de enfermagem (COFEN). Resolução $n^{\circ}$ 258/2001. Rio de Janeiro: Coren-RJ; 2001 Jul 12 [acesso em 2010 mar 25]. Disponível em:

http://site.portalcofen.gov.br/node/4296

17. Oliveira ELF, Morais JMC, Olímpio NO, Baptista I. Principais indicações para o uso do cateter central de inserção periférica (PICC): Fatores limitantes. 2006 [cited 2010 Out 26]; Available from: http://www.inicepg.univap.br/cd/INIC 2006/inic/inic/03/INIC000054 6_OK.pdf

18. Bardin L. Análise de conteúdo. Lisboa: Edições 70, 2011. 279 p. 19. Câmara SMC, Tavares TJL, Chaves EMC. Cateter Venoso de Inserção Periférica: análise do uso em recém nascidos de uma unidade neonatal pública em Fortaleza. Rev. Rene. 2007; 8(1):32-37.

Artigo recebido em 12/01/2011.

Aprovado para publicação em 01/02/2012.

Artigo publicado em 31/12/2012. 